Finally, the virus-like particles most commonly found in human milks bear no resemblance to mouse mammary tumour virus B-type particles but do resemble mouse leukaemia virus particles. In short, it seems that no great significance can be attached to the structure of the various virus-like particles which can be isolated from human milks.

But what about the comparative distribution of these various virus-like particles in the milks of women from families with or without a history of breast cancer? Last year Moore et al. reported that B-type particles (structures which they would now classify either as MS-1 or MS-2 particles) were present in the milk of six out of ten American women belonging to families with a history of the disease, but only seven of the milks from 156 women belonging to families without a history of the disease contained particles. Moreover, they also reported that Btype particles were present in eighteen of the milks from forty-six Parsi women. There seemed to be a strong positive correlation between the occurrence of the B-type particles and an increased risk to an incidence of breast cancer but, now a larger number of milks has been screened, that correlation no longer applies. The data given by Moore et al. in their first table (page 105) indicate that there is no significant difference between the distribution of any of the three types of virus-like particles which they identify in milks from women belonging to families with or without a history of the disease.

One has only to read the last paragraph of what Moore et al. have to say in this issue of Nature and then immediately re-read the last paragraph of their report published last year to realize the extent to which doubts and uncertainties have replaced confident enthusiasm about the role of putative viruses in human breast cancer.-From our Cell Biology Correspondent.

\section{CYCLIC AMP}

\section{Role in Higher Plants}

from our Plant Physiology Correspondent THE action of cyclic $3^{\prime}, 5^{\prime}$-adenosine monophosphate (cyclic AMP) as the mediator of the action of several mammalian hormones has been established for some years. Similarly, its activity as an important intermediary in the regulation of gene expression in certain microorganisms is now recognized. Consequently, observers with a feeling for the unity of biochemistry will doubtless have been relieved by the recent flush of articles reporting a possible role for cyclic AMP in the third major group in the biosphere, the green plants.

Latest among these reports is the finding by Henry Wood, Michael Lin and Armin Braun, at Rockefeller University, that a cell division factor, isolated from crown gall tumour tissues and chemically, though apparently not physiologically, closely related to the cytokinin hormones of healthy plants, is a potent inhibitor of cyclic AMP phosphodiesterase (Proc. US Nat. Acad. Sci., 69, 403; 1972). This enzyme catalyses the conversion of cyclic AMP to 5'-AMP and thus could operate to regulate the active levels of cyclic AMP within the cells. If cyclic AMP is postulated to be essential for cell division, the promotion of cell division by the extracted factor can be easily understood. The cell division factor is equally effective on bovine brain phosphodiesterase and on enzyme prepared from the crown gall tissues. Furthermore, Wood et al. report that adenyl cyclase, an enzyme which catalyses the formation of cyclic AMP from ATP, can be detected as a membrane-bound component in the tumour tissues.

The first indication that cyclic AMP may have a role in hormone action in plants came from the finding that the nucleotide could replace the hormone gibberellic acid in the induction of $\alpha$-amylase synthesis in barley aleurone layers, tissues that store starch in the seed (Duffus and Duffus, Experientia,
25. 581; 1969; Galsky and Lippincott, Pl. Cell Physiol., 10, 607; 1969). Pollard (Biochim. Biophys. Acta, 201, 511; 1970) was the first to obtain evidence for the formation of cyclic AMP in higher plants when he demonstrated the conversion of ${ }^{14} \mathrm{C}$-adenine into cyclic AMP by isolated aleurone layers. The rate of formation of cyclic AMP was doubled in aleurone layers which had been treated with gibberellic acid. Last year, using a similar approach, Saloman and Mascarenhas showed that auxin treatment of oat coleoptiles caused a 50-80 per cent increase in formation of cyclic AMP from exogenous adenine (Life Sci., 10, 879; 1971). Thus, although much remains to be done on, for example, the questions of specificity of action, and the magnitude and rapidity of the responses, evidence seems to be accumulating that cyclic AMP may be acting in plants, as in mammals, as an intracellular second messenger for auxins, gibberellins, and at least close relatives of the cytokinins.

From the point of view of the plant physiologist, of course, this is an exciting new development and it is likely that many related investigations have already begun in other laboratories in response to these findings. Nevertheless, it is somewhat saddening that the study of cyclic AMP in higher plants should have lagged so many years behind the exciting discoveries in microorganisms and higher animals.

\section{New Light on Folded DNA}

Nor long ago Lerman reported that linear double-helical DNA could undergo a transition to a compact non-disymmetric form in solutions containing neutral or acidic polymers and relatively high salt concentrations. Jordan, Lerman and Venable in next Wednesday's Nature New Biology (March 22) present a further investigation of this compact form (or $\Psi$-DNA, as they term it) by circular dichroism.

The principal conclusion is that the $\Psi$-DNA is characterized by a higher level of order, and not by any change in the geometry of the Watson-Crick helix. The formation of the $\Psi$-form is a process requiring some hours, and is accompanied by a gross change in the circular dichroism, the usual positive extremum in the long-wavelength region of the absorption band being completely eliminated. The appearance of this new form follows first-order kinetics. In due course the circular dichroism undergoes a further change, with a large enhancement in negative ellipticity $\left(\Psi^{*}\right.$-form). The magnitude of the effect reaches a limiting value with neutral polymer (polyethylene oxide) concentration and with ionic strength. In the initial formation of the $\Psi$-form, there is a sharp extremum in the profile of ellipticity against polymer and against salt concentration. This is evidently a kinetic effect only.

Jordan et al. explain the phenomenon in terms of the formation of nuclei for the growth of the ordered compact form: the conditions best favouring nucleation being different from those which correspond to maximum stability of the completed structure. The most likely mechanism involves the initial formation of an extended form of the DNA, which is then able to enter the new ordered compact state. X-ray diffraction indicates that the primary helix remains in the B-form.

The circular dichroism spectra resemble those of DNA in various forms, such as complexes with basic polymers, in which it is also presumed to be in a compact, condensed state. The form of the circular dichroism spectra is probably governed by inter-helix interactions. The DNA evidently does not interact directly with the neutral polymer, nor is the concentration of the latter sufficient to effect a large change in water activity. The thermodynamic reasons for the formation of the $\Psi$-form thus remain obscure. 